\section{Dynamic Parathyroid CT: Are 2 Phases Sufficient?}

We read with interest the recent case series by Gafton et al, ${ }^{1}$ sharing their experience with dual-phase CT of the neck for detection of parathyroid hormone (PTH)-secreting lesions. To date, much of the literature on multiphasic CT for detection of parathyroid adenoma has focused on the overall diagnostic performance of the technique and less on the specific features of PTH-secreting lesions that differentiate them from normal tissue. ${ }^{2-4}$ In conjunction with recently published data by Beland et $\mathrm{al}^{5}{ }^{5}$ the bar graph depicting mean enhancement values for parathyroid hormone-secreting lesions, thyroid tissue, lymph nodes, and submandibular glands is a welcome step toward establishing criteria that are predictive of a PTH-secreting lesion.

We wish to make an observation regarding the data presented in this article, as to how they differ from our experience and how this difference may impact clinical scanning protocols.

Gafton et $\mathrm{al}^{1}$ noted that all tissue other than PTH-secreting lesions accumulated contrast between the arterial and venous phases. Our experience has been different. We typically note a mild degree of contrast washout in thyroid tissue between the arterial and venous phases. This is likely related to slight differences in scan timing. The protocol described by Gafton et al begins scanning at 25 seconds after the initiation of a $120-\mathrm{mL}$ contrast bolus delivered at $3 \mathrm{~mL} / \mathrm{s}$. Given the speed at which a 64-row multidetector scanner covers the imaged volume, it is possible that the thyroid gland had not yet reached peak enhancement during the arterial phase imaging in their series.

At our institution, the arterial phase scan is performed 30 seconds after the initiation of a $120-\mathrm{mL}$ contrast bolus delivered at a rate of 4 $\mathrm{mL} / \mathrm{s}$, and the venous phase is performed at a 60 -second delay. This slight difference in timing leads to a reversal of the arterial-to-venous attenuation relationship in thyroid tissue, which can obscure a PTHsecreting lesion adjacent to the thyroid gland. This issue is easily resolved by looking at the precontrast images, which we often find very useful.

While we agree wholeheartedly with efforts to reduce medical radiation to the minimum necessary levels, we believe that the optimal protocol for dynamic parathyroid CT remains an active question.

\section{References}

1. Gafton AR, Glastonbury CM, Eastwood JD, et al. Parathyroid lesions: characterization with dual-phase arterial and venous enhanced CT of the neck. AJNR Am J Neuroradiol 2012 Jan 12. [Epub ahead of print]

2. Rodgers SE, Hunter GJ, Hamberg LM, et al. Improved preoperative planning for directed parathyroidectomy with 4-dimensional computed tomography. Surgery 2006;140:932-40, discussion 940-41

3. Harari A, Zarnegar R, Lee J, et al. Computed tomography can guide focused exploration in select patients with primary hyperparathyroidism and negative sestamibi scanning. Surgery 2008;144:970-77

4. Kutler DI, Moquete R, Kazam E, et al. Parathyroid localization with modified 4D-computed tomography and ultrasonography for patients with primary hyperparathyroidism. Laryngoscope 2011;121:1219-24

5. Beland MD, Mayo-Smith WW, Grand DJ, et al. Dynamic MDCT for localization of occult parathyroid adenomas in 26 patients with primary hyperparathyroidism. AJR Am J Roentgenol 2011;196:61-65

A.R Sepahdari Department of Radiological Sciences

A. Harari

Department of General Surgery David Geffen School of Medicine University of California Los Angeles Los Angeles, California 